\title{
Conocimiento del profesor acerca de las características de aprendizaje del álgebra en bachillerato
}

\section{Teacher Knowledge about the features of learning algebra in high school}

\author{
Leticia Sosa \\ Universidad Autónoma de Zacatecas \\ lsosa@mate.reduaz.mx \\ Eric Flores-Medrano, José Carrillo \\ Centro de Investigación en didácticas especificas e investigación en el aula (CIDIESIA), Universidad de Huelva \\ ericfm_0@hotmail.com, carrillo@uhu.es
}

RESUMEN • El objetivo de este estudio es avanzar en la caracterización de uno de los subdominios del conocimiento didáctico del contenido, en concreto del conocimiento de las características del aprendizaje de las matemáticas. Con un enfoque interpretativo, se desarrolla un estudio de caso con dos profesoras de segundo de bachillerato en el cual el contexto matemático está centrado en álgebra lineal. Se aporta un sistema de categorías construido a partir de indicadores basados en literatura especializada y en datos empíricos propios de esta investigación.

PALABRAS CLAVE: conocimiento de las características de aprendizaje de las matemáticas; conocimiento didáctico del contenido; conocimiento especializado del profesor de matemáticas.

ABSTRACT - The aim of this study is to improve the characterization of one of the subdomains of pedagogical content knowledge, namely knowledge of the features of learning mathematics. With an interpretive approach, we developed a case study with two high school teachers, the content being focused on Linear Algebra. Categories and indicators to deepen this characterization, derived from research literature and from empirical data from this study, are reported.

KEYWORDS: knowledge of features of learning mathematics; pedagogical content knowledge; mathematics teacher's specialised knowledge. 


\section{INTRODUCCIÓN}

Desde hace treinta años, y cada vez más, se ha venido discutiendo y profundizando en el estudio del conocimiento profesional de los profesores (Varas, Lacourly, López y Giaconi, 2013). El trabajo de Shulman (1986) se reconoce como pionero en llamar la atención sobre el carácter específico del conocimiento necesario para enseñar y su propuesta ha jugado un papel importante en el desarrollo de investigaciones e implementaciones curriculares para la formación de profesores.

Un elemento que habitualmente es considerado en diversos modelos de conocimiento del profesor de matemáticas es el referente al sujeto cognoscente y al propio proceso de aprendizaje. En particular, en este artículo nos posicionaremos en el modelo MTSK (Mathematics Teacher's Specialised Knowledge) para estudiar en profundidad el conocimiento de las características de aprendizaje de las matemáticas. El estudio de este conocimiento cobra relevancia ya que permite entender, entre otras cosas, qué elementos requiere el profesor para anticiparse a los modos de pensamiento del estudiante, cómo interpreta sus producciones y lenguaje matemático, así como la manera en la que identifica, aprovecha y devuelve las oportunidades de aprendizaje que surgen a partir de la actividad matemática de los estudiantes. Pretendemos identificar, conocer y comprender indicadores sobre el conocimiento de las características de aprendizaje de las matemáticas surgidos de la literatura de investigación y de la práctica de enseñanza (más que evaluar si el conocimiento del profesor es correcto o no). Esto proporciona, además de un acercamiento a la comprensión de la complejidad del conocimiento didáctico del contenido (Pinto y González, 2006), una aportación pues atiende la limitación de que los modelos teóricos del conocimiento matemático para la enseñanza suelen incluir categorías muy generales (Godino, 2009).

\section{FUNDAMENTOS TEÓRICOS}

Shulman (1986) considera que los tres componentes fundamentales que sostienen la especificidad de cada materia a enseñar son: conocimiento del contenido, conocimiento didáctico del contenido (PCK) y conocimiento curricular. El PCK incorpora aspectos relacionados a la enseñanza y al aprendizaje del contenido y da entidad a un cuerpo específico de conocimiento para el profesor. Señala que el PCK incluye «la comprensión de lo que hace el aprendizaje de temas específicos fácil o difícil: las concepciones y preconcepciones que los estudiantes de diferentes edades y procedencias traen para el aprendizaje de los temas y lecciones frecuentemente más enseñadas» (p. 9). Cabe mencionar que, como expresan Ponte y Chapman (2006), Shulman hizo hincapié en el PCK como un aspecto clave para abordar en el estudio de la enseñanza, y es precisamente éste el dominio que ha sido el foco de atención en muchas investigaciones acerca del conocimiento profesional del profesor (Even y Markovits, 1991; Hill, Ball y Schilling, 2008).

Un modelo que en su estructura considera elementos relacionados con el PCK es el MTSK (figura 1). Parte de la reflexión sobre cuál es el conocimiento que solo tiene sentido para el profesor de matemáticas y hace una llamada de atención sobre la importancia de lo especializado del conocimiento del profesor de matemáticas, entendiendo esta especialización como un conjunto de seis subdominios de naturaleza diferenciable (Carrillo, Climent, Contreras y Muñoz-Catalán 2013). 


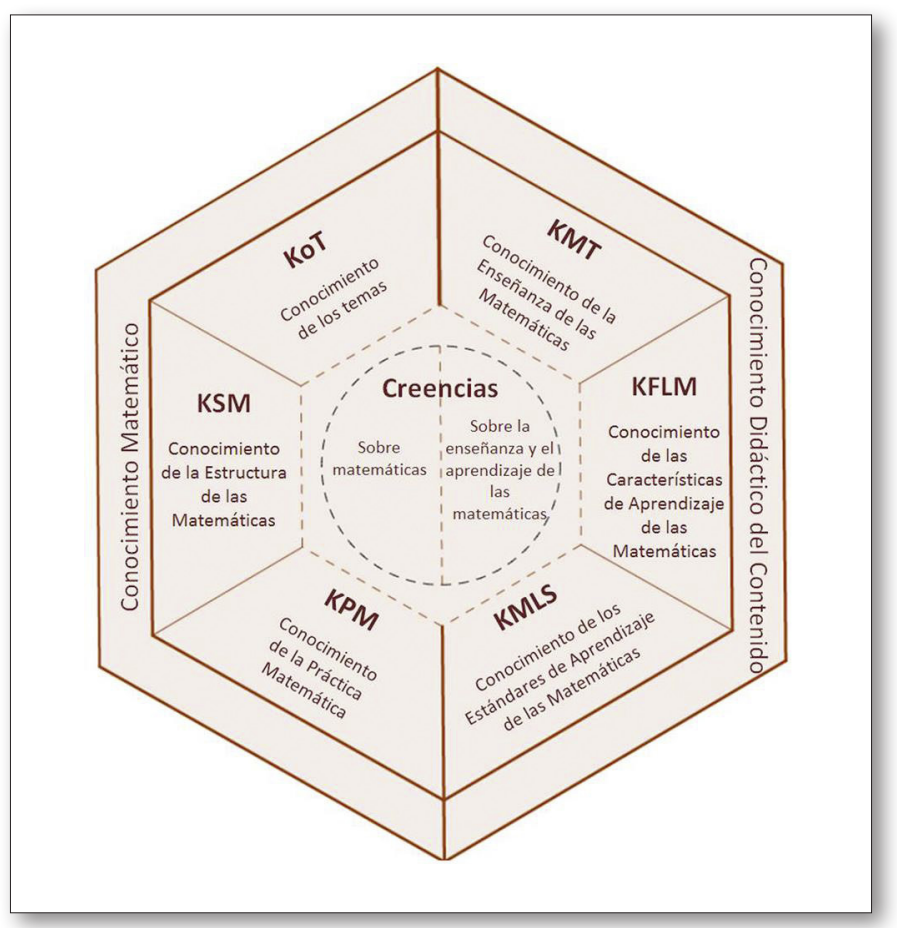

Fig. 1. Subdominios del MTSK. ${ }^{1}$

De manera sucinta presentamos a continuación los subdominios del MTSK. Interesa especialmente, como foco de este artículo, el conocimiento de las características del aprendizaje en matemáticas, que es parte del PCK.

En el dominio del conocimiento matemático encontramos el conocimiento de los temas (conocimiento de conceptos, aspectos fenomenológicos, significados, definiciones, etc., que caractericen aspectos del tema abordado), el conocimiento de la estructura matemática (conocimiento de cómo se dan las conexiones entre temas, de la matemática elemental desde un punto de vista avanzado y de la matemática avanzada desde un punto de vista elemental) y el conocimiento de la práctica matemática (conocimiento de las formas de proceder, conocer y crear en matemáticas).

En el dominio del conocimiento didáctico del contenido (PCK) se contempla el conocimiento de los estándares de aprendizaje de las matemáticas (conocimiento acerca de lo que el estudiante debe/ puede alcanzar en un curso escolar determinado, sobre las capacidades conceptuales, procedimentales y de razonamiento matemático que se promueve en determinados momentos educativos), el conocimiento de la enseñanza de las matemáticas (conocimiento de recursos materiales y virtuales, modos de presentar el contenido y el potencial que puede tener para la instrucción, así como el conocimiento de ejemplos adecuados para cada contenido) y el conocimiento de las características de aprendizaje de las matemáticas (conocimiento que tiene el profesor acerca del contenido matemático como objeto de aprendizaje, en lugar de poner en el centro el conocimiento sobre el estudiante se pone el proceso de aprendizaje normado por el contenido matemático). El estudio de este último se verá fortalecido por la revisión de literatura presentada en la siguiente sección y por el análisis de los datos empíricos que reportamos en este artículo.

1. Se emplean las siglas de los nombres de la versión en lengua inglesa del modelo. 
Además, en el modelo se consideran las concepciones que tiene el profesor acerca de las matemáticas, su enseñanza y aprendizaje. Estas concepciones se encuentran en el centro del modelo y están delimitadas con líneas punteadas para reflejar que las consideramos como agentes que permean a cada uno de los elementos de conocimiento involucrados en el modelo y que es pertinente el estudio de las relaciones entre dichas concepciones y conocimientos por formar parte, respectivamente, de la estructura afectiva y cognitiva del profesor.

\section{CONOCIMIENTO DE LAS CARACTERÍSTICAS DE APRENDIZAJE DE LAS MATEMÁTICAS: ESTUDIOS PREVIOS}

El conocimiento de las características de aprendizaje de las matemáticas es derivado de la necesidad de que el profesor conozca y entienda lo que pueden pensar matemáticamente los estudiantes ante las actividades que se le asignan. Considera específicamente los conocimientos que están normados por el contenido matemático, centrando así su atención en el conocimiento que tiene el profesor acerca de los procesos de aprehensión de los objetos matemáticos y de los fenómenos derivados de esto (errores comunes, obstáculos epistemológicos, estructuras mentales asociadas a contenidos particulares, etc.).

Los estudios que tratan sobre el conocimiento del profesor de matemáticas habitualmente consideran lo relacionado con el estudiante como sujeto cognoscente o el conocimiento que le permite identificar que una actividad matemática es realmente producida por el estudiante (Kuzniak, 2011), esto último relacionado con el proceso de aprendizaje. Es notoria la influencia que dejan los trabajos de Shulman, en particular la introducción del Pedagogical Content Knowledge, en diversos modelos que conceptualizan este tipo de conocimiento. También apoyados en el PCK, desde una visión transformativa en términos de Gess-Newsome (1999), Silverman y Thompson (2008) adoptan un modelo de desarrollo de conocimiento para la enseñanza de las matemáticas con características que, de acuerdo con su perspectiva, dan cuenta del desarrollo que le permite a un profesor sustentar una enseñanza conceptual de un contenido determinado. De estas características, las que se relacionan con el estudiante o con su aprendizaje de las matemáticas consideran que el profesor debe poseer un conocimiento que le permita anticiparse a los modos de pensamiento de los estudiantes en un tema determinado y de las formas en que se puede guiar dicho pensamiento a uno idóneo y cómo este le ayudará a integrar o enfrentarse a nuevos contenidos matemáticos.

En el marco del Teacher Education and Develompment Study in Mathematics, Tatto et al. (2008) conforman un marco conceptual en el que, para el PCK, consideran tres categorías: el Mathematical curricular knowledge, el Knowledge of planning for mathematics teaching and learning y el Enacting mathematics for teaching and learning. Los aspectos relacionados con el estudiante o su aprendizaje de matemáticas son predecir respuestas típicas de estudiantes, incluyendo concepciones erróneas; analizar o evaluar soluciones y argumentos matemáticos de los estudiantes; analizar el contenido de las preguntas de los estudiantes, y diagnosticar respuestas típicas de los estudiantes, incluyendo las concepciones erróneas.

Por otro lado, Kilpatrick, Swafford y Findell (2001) presentan un modelo sobre aquello que consideran que da muestras del aprendizaje de los estudiantes sobre diversos contenidos. Proponen el término Proficiency que se entiende como el conjunto de habilidades, conocimientos y actitudes necesarias para aprender matemáticas. En este modelo consideran cinco hebras que son retomadas en Flores-Medrano, Escudero-Avila, Montes y Carrillo (2014) para el desarrollo de una categorización del conocimiento que tiene el profesor sobre el aprendizaje de las matemáticas. Esta considera el conocimiento de la comprensión conceptual de contenidos, de la fluidez procedimental de los estudiantes, de las estrategias de abordar problemas matemáticos, de las formas de razonar y de las actitudes de los estudiantes frente a las matemáticas. 
Con base en un modelo epistemológico de corte antropológico, Godino (2009) propone un modelo de facetas y niveles del conocimiento del profesor que incluye categorías y componentes tanto del conocimiento de contenido matemático como del didáctico. Los aspectos de conocimiento del profesor relacionados directamente con los estudiantes o su aprendizaje de las matemáticas están en las facetas cognitiva [«conocimientos personales de los estudiantes y progresión de los aprendizajes» (p. 21)], afectiva [«estados afectivos (actitudes, emociones, creencias, valores) de cada alumno con relación a los objetos matemáticos y al proceso de estudio seguido» (p. 21)] y la interaccional ["patrones de interacción entre el profesor y los estudiantes y su secuenciación orientada a la fijación y negociación de significados» (p. 21)]. Estas facetas interactúan con el resto (epistémica, mediacional y ecológica), pero su separación responde a una necesidad de identificación de cada faceta en los diferentes estudios.

Como parte del desarrollo del modelo MKT, Hill, Ball y Schilling (2008) realizan un estudio para conceptualizar y medir el conocimiento de los profesores en cuanto a los estudiantes y contenidos específicos en el cual exponen cuatro categorías: errores comunes de los estudiantes (identificar y proporcionar explicaciones sobre errores y conocer qué errores se presentan con qué contenido), comprensión de lo que hacen los estudiantes (interpretar qué producciones de los estudiantes son suficientes y mejores para mostrar comprensión), las secuencias del desarrollo de los estudiantes (identificar los tipos de problemas, temas o actividades matemáticas que son más fáciles o más difíciles a determinada edad, sabiendo lo que los estudiantes aprenden típicamente primero) y estrategias comunes de cálculo de los estudiantes (familiarización con los números y las operaciones). En el trabajo de Rowland et al. (2005) también podemos encontrar información respecto a lo que esperan que un profesor conozca sobre el aprendizaje de los estudiantes, cuando presentan las cuatro categorías del Knowledge Quartet (fundamentos, transformaciones, conexiones y contingencias), por ejemplo el código de identificación de errores en la categoría de fundamentos y el de enfrentar y resolver concepciones erróneas comunes en la de transformaciones, además consideran las dificultades de los alumnos al explicar de manera más detallada la lección de una profesora. Finalmente, Ma (2010), en el desarrollo del modelo de comprensión profunda de la matemática, aborda aspectos de profesores sobre el manejo del error de los alumnos (identificar y explicar cómo ayudarían a los alumnos a corregir el error) ante el contenido de la multiplicación de números con varios dígitos, considerando también en ello lo relacionado con las confusiones de los alumnos.

En síntesis, de esta revisión podemos extraer que se espera que los profesores de matemáticas tengan conocimiento sobre las concepciones y conocimientos previos de los estudiantes (en algunos casos se incluye el conocimiento de cómo estos conocimientos potencian el aprendizaje de nuevos contenidos); de las dificultades al enfrentarse a diversos contenidos o a la matemática en sí (puede incluir también lo que resulta fácil de aprender); de la variedad de formas o procesos con los que el estudiante puede comprender el contenido (se considera el conocimiento que le permite al profesor discernir aquellas formas suficientes y también las más efectivas); de las concepciones de este frente a las matemáticas; de preguntas y respuestas típicas de los estudiantes (está incluido aquí el conocimiento sobre las concepciones erróneas y los errores más comunes), y de las formas en las que estos resuelven y se enfrentan a problemas matemáticos.

\section{DISEÑO DE LA INVESTIGACIÓN}

Esta investigación es de corte interpretativo (Latorre, Rincón y Arnal, 1996) y se emplean métodos cualitativos (Merriam, 1988), ya que nuestro propósito es comprender e interpretar el Conocimiento de las Características de Aprendizaje de las Matemáticas (KFLM), evidenciado por dos profesoras al impartir álgebra en bachillerato. También tomamos esa decisión por la riqueza holística de los estudios 
cualitativos en cuanto a mirar el contexto en su forma natural a partir de sus distintos ángulos y perspectivas (Bisquerra, 2004).

\section{LOS CASOS}

El diseño de la investigación es un estudio de dos casos (Emi y Aly) de tipo instrumental (Stake, 1994). Esta clase de estudio permite profundizar en la comprensión de un tema determinado o afinar una teoría. En este estudio pretendemos tener una mejor comprensión del KFLM en bachillerato, siendo el álgebra lineal el contexto matemático en el cual se desarrollan los dos casos. Emi y Aly son licenciadas en Matemáticas e imparten álgebra en segundo año de bachillerato. En el momento de realizar la investigación, Emi cuenta con veintiún años de experiencia y enseña a estudiantes del bachillerato de Ciencias Sociales; mientras que Aly lo hace a estudiantes del bachillerato Científico Tecnológico y tiene trece ańos de experiencia.

\section{COLECCIÓN DE DATOS Y ANÁLISIS}

Esta investigación es parte de un estudio más amplio en el que, en concordancia con Yin (1984), intentamos sacar todo el provecho posible desde múltiples fuentes de evidencia, combinamos varios instrumentos para la recogida de la información: observaciones de aula, notas de campo, cuestionarios y entrevista semiestructurada.

Las observaciones de aula son la fuente de información principal para este estudio. Corresponden a las clases impartidas por las dos profesoras al comienzo del curso utilizando el método de observación no participante (Cohen y Manion, 2002). Se filmaron 15 clases de cada profesora, con una duración aproximada de 50 minutos cada una. Recogimos notas de campo en el transcurso de las clases impartidas, porque con estas se obtienen impresiones que complementan la observación, pues, de acuerdo con Evertson y Green (1989), son registros que incluyen aspectos teóricos, puntos de vista y reflexiones personales que subyacen en la observación de clases; las tomamos in situ y a posteriori, como sugieren Lofland y Lofland (1984).

Después de la observación de clases se realizaron seis cuestionarios y una entrevista semiestructurada a cada una de las profesoras para comprender lo evidenciado en las observaciones de aula.

El análisis de los datos tuvo por objetivo obtener indicadores del KFLM para Emi y Aly. Partimos de la extracción de episodios de las diferentes clases observadas. Dicha elección se valió de la sensibilidad teórica que nos brindó la revisión de la literatura. Cabe señalar que no comenzamos con categorías establecidas, sino que la revisión de literatura nos ayudó a afinar lo que entendemos en cuanto a la naturaleza del Conocimiento de las Características de Aprendizaje de las Matemáticas y algunos fenómenos en los cuales se refleja dicho conocimiento. Una vez elegidos los episodios realizamos un proceso de abstracción de indicadores de conocimiento. Estos indicadores fueron agrupados por similitud entre ellos para someterlos a un segundo proceso de abstracción, lo cual dio como resultado la generación de categorías para el KFLM.

\section{RESULTADOS}

En esta sección presentamos episodios (seleccionados a partir de la sensibilidad teórica) y cómo una abstracción sobre estos nos permitió generar indicadores de conocimiento para el subdominio KFLM. La presentación de los indicadores no responde al orden cronológico de las clases de las dos profesoras sino a la agrupación por similitud de estos que, en un segundo proceso de abstracción, nos genera las categorías que son resultado de este artículo. 
La clase 15 de Aly es sobre resolución de ejercicios acerca de sistemas homogéneos. Uno de ellos consiste en un sistema $(x+y+5 z=0,3 x-y-2 t=0, x-y+z-t=0)$ en el que hay que averiguar a través del rango si es compatible o no, y en caso de serlo decir de qué tipo.

Aly: Fijaos que ahí tenemos un sistema homogéneo, ¿̇de cuántas ecuaciones y de cuántas incógnitas estamos hablando ahora? [...]

E1: Tres ecuaciones y cuatro incógnitas [...] Una pregunta, ¿eso no es incompatible?

Aly: No, no es incompatible, hay que hacerlo.

E1: No, pero el rango va a ser menor que el número de incógnitas.

Aly: Pero aunque sea rango uno o rango dos, incompatible no va a serlo nunca.

E1: Ah, vale, vale.

Aly: Si es un sistema homogéneo, como ese, nunca te va a salir incompatible, siempre va a ser compatible, lo que tienes que ver es si es compatible determinado o indeterminado. Aquí lo que tú estás intentando decir es que va a salir compatible indeterminado, seguro.

E1: Sí.

Aly: Claro, pero compatible, ¿vale?

En este episodio se evidencia que Aly utiliza su conocimiento matemático sobre sistemas homogéneos para interpretar una respuesta dada por uno de sus estudiantes (ella sabe qué significa sistema (in)compatible y las condiciones para que se dé, por eso no admite como válida la respuesta que da el estudiante). Interpreta que lo que intenta decir E1 es que el sistema homogéneo va a salir compatible indeterminado porque sabe también que para un estudiante que tiene un primer acercamiento a este tópico matemático en su lenguaje usa como indistintas las palabras incompatible o indeterminado, en parte porque apenas está construyendo el significado matemático de estas palabras en los sistemas homogéneos. Por otro lado, en la primera clase grabada de Emi, cuando ella les pregunta a sus estudiantes qué recuerdan de la clase pasada en la que se introdujo el tema de matriz, un estudiante contesta que "había que poner los datos en una especie como de tabla», para referirse a la introducción de los coeficientes de los sistemas de ecuaciones en una matriz. En la segunda clase, antes de introducir distintos tipos de matrices, Emi vuelve a preguntar el significado de matriz y obtiene por respuesta: «como una tabla con números, es un conjunto ordenado de datos de un problema», a lo que ella comenta: "podemos definir matriz como un conjunto de elementos ordenados dispuestos en filas y en columnas». Estamos hablando de respuestas en las que el estudiante pone en juego su lenguaje común mientras adquiere el lenguaje matemático adecuado, y en las que el estudiante responde mezclando lenguaje común con lenguaje matemático. De esta forma queremos destacar esa mezcla de lenguaje que usa el estudiante al estar construyendo y tratando de hacer suyo un contenido matemático que para él es nuevo. El estudiante la usa porque está aprendiendo, incluso, a familiarizarse con el lenguaje matemático de ese contenido. En este sentido, pretendemos hacer notar el conocimiento del profesor que le permite saber interpretar el conocimiento/pensamiento matemático que expresan los estudiantes a través de su lenguaje. Así, extraemos el indicador:

KFLM1. Saber interpretar el conocimiento o pensamiento matemático que expresan los estudiantes en su lenguaje (común o en proceso de adquisición del nuevo contenido matemático -mezcla del lenguaje común con el matemático).

En la clase grabada 15 de Aly, al hacer un ejercicio que trata del sistema homogéneo $x-y-z=0$, $x+y+3 z=0, x-5 y-9 z=0$, les explica que el rango es, como mínimo, 1 (porque hay un menor de orden 1 dado que el primer elemento, de la primera fila y primera columna es distinto de 0) y luego que al menos es 2 (si se amplía con la segunda columna y la segunda fila y se toma la submatriz de orden 2). Para ver si el rango es 3, les indica que hay que calcular el determinante $3 \times 3$ de la matriz de coeficientes, que da cero y que por tanto el rango de la matriz es 2 , con lo cual se trata de un sistema compatible indeterminado. Añade: 
Entonces la tercera ecuación sobra porque habrá una dependencia lineal entre dos filas, que sea fácil de ver o no, no me preocupa, el caso es que tiene que existir dado que el determinante me ha dado 0 , ¿̇vale?

Aly sabe que puede haber estudiantes a los que les pueda interesar averiguar la dependencia lineal y no llegar a resolver el problema (ni a encontrar la dependencia lineal); ante eso realiza hace el siguiente comentario: "que sea fácil de ver o no, no me preocupa». A ella le interesa que los estudiantes no se desvíen y terminen de resolver el problema. Normalmente, cuando el profesor propone un ejercicio o problema que resolver lo hace con una intencionalidad. Al resolver el problema previamente puede conocer las estrategias de solución que pueden seguir los estudiantes (Kilpatrick et al., 2001) y los detalles en los que pueden desviarse matemáticamente y perder el sentido del problema, es decir, aspectos que puedan desorientar la intencionalidad de ese problema o ejercicio y que por ello no logren llegar a la solución. Podemos decir que esto está relacionado con el conocimiento del profesor referente a las producciones suficientes (y más adecuadas) para comprender un contenido matemático (Hill et al., 2008). De esto abstraemos el indicador:

KFLM2. Conocer los detalles de la resolución de un problema susceptibles de desviar la atención de los estudiantes para llegar a la solución del mismo.

Destacando así el conocimiento del profesor referente a los atascos, despistes o desvíos cognitivos que pueden presentar los estudiantes para poder dar solución a un problema matemático.

Ahora presentamos el caso de Aly (en la clase grabada 14), en relación con un problema de matrices donde existe la necesidad de despejar $X$ de $A X=C$. Aly sabe que los estudiantes pudieran equivocarse al multiplicar por $\mathrm{A}^{-1}$ (cuando exista la matriz inversa de A) sin detenerse a pensar si debe ser por la derecha o por la izquierda de $\mathrm{C}$, o sea, Aly sabe cuándo los estudiantes pueden ejecutar procedimientos sin saber por qué o para qué.

Aly: Es decir, el sistema generalmente lo escribimos así $A X=C$, ¿̇verdad? Entonces ayudándonos ahora de la matriz inversa, ¿cómo despejaríamos la $X$ (que verdaderamente son las incógnitas)?

E1: Pues multiplicando por la inversa del lado donde no está la $X$.

Aly: Si multiplicamos por la inversa, tenéis que tener cuidado, o multiplico por la izquierda o multiplico por la derecha, no puedo multiplicar de cualquier manera puesto que el producto no es conmutativo; en este caso, para que la $\mathrm{A}$ a la menos uno se quede al lado de la $A$ y nos quede la matriz identidad, ¿por qué parte tengo que multiplicar, por la derecha o por la izquierda?

E1: Por la izquierda.

Aly: Por la izquierda, es decir, multiplicaríamos por $A^{-1}$ [escribe $A^{-1} A X=A^{-1} C$ ]. Fijaos en la segunda parte de la igualdad, aquí es lo importante, pues si multiplicamos por la izquierda por $A^{-1}$ de un lado de la igualdad, también lo tenemos que hacer por la izquierda en la segunda parte de la igualdad, pues no es lo mismo $A^{-1}$ por $\mathrm{C}$ que $\mathrm{C}$ por $A^{-1}$, son dos operaciones distintas.

También podemos hablar de la clase grabada 8 de Emi, donde se muestra que un estudiante piensa que al obtener el valor de $x, y, z$ está obteniendo tres soluciones al sistema, sin tomar conciencia de que el valor de esas tres variables constituye una solución.

Emi: ¿Cuántas soluciones nos han salido en el problema?

E10: Tres.

Emi: Cuando ponemos $x=, y=, z=$, ¿cuántas soluciones estamos dando?

E10: Tres.

Emi: Por ejemplo $x=10, y=50$ y $z=30$, ¿cuántas soluciones?

E3: Una.

Emi: Es una solución, es decir, son tres incógnitas, pero es una solución. Porque el sistema está formado por varias ecuaciones y en este caso tenemos nosotros tres incógnitas, hallar la solución es hallar el valor para cada una de las incógnitas, si tenemos un valor para cada una de las incógnitas, pues tenemos sólo una solución, ¿̨de acuerdo? 
En el transcurso de la clase, el estudiante realiza varios cálculos matemáticos mecánicamente, por ello es relevante que el profesor conozca eso, a fin de ayudarlo a tomar conciencia de lo que está haciendo matemáticamente (por qué y para qué), con el propósito de provocar la reflexión del estudiante e intervenir en la orientación de su aprendizaje matemático.

Cabe mencionar que existen varios autores que consideran importante el conocimiento del profesor en cuanto a la variedad de formas o procesos con los que el estudiante puede comprender un contenido (Silverman y Thompson, 2008). En este caso, las evidencias nos permiten ver un aspecto más concreto relacionado con lo que los estudiantes pueden hacer mecánicamente, por ello proponemos el indicador:

KFLM3. Conocer los cálculos matemáticos que podrían hacer mecánicamente los estudiantes sin saber realmente lo que están haciendo matemáticamente.

Estamos hablando de un conocimiento del profesor que podría permitirle emplear medidas preventivas y discernir si el estudiante ha comprendido el contenido matemático que pretende enseñarle o solo ejecuta procedimientos o cálculos matemáticos sin saber por qué o para qué.

Con estos tres indicadores intentamos dar cuenta de aspectos del conocimiento del profesor en cuanto a las características de aprendizaje asociadas más concretamente con el lenguaje que usan los estudiantes al aprender un contenido y con procesos que los estudiantes desarrollan al relacionarse con un contenido matemático; por eso hemos etiquetado la primera categoría como: lenguaje y procesos con los que los estudiantes interactúan con el contenido.

En el siguiente episodio (clase grabada 11), Emi va a impartir el tema de programación lineal y prevé que a E2, por su deficiencia visual, le costará mucho trabajo comprender los problemas, debido a que habrá dibujos y gráficas. Emi sabe que E2 tiene buena capacidad de aprendizaje pero reconoce que ella, como profesora, requiere proveerla de apoyos para impulsar su aprendizaje.

En este libro de texto, debido a la representación gráfica, E2, que hoy no está en clase, precisamente hasta ahora yo le había dado las matemáticas como a vosotros, ¿no?, con un tamańo de letra adecuado para que ella más o menos captase las cosas, luego ya con la memoria que tiene y con su capacidad pues es capaz de reproducirlo y de resolverlo, pero todos estos dibujos, todas estas gráficas que hay que realizar, necesito el apoyo del papel para que ella entienda lo que estoy explicando porque todo lo que se pone en la pizarra ella no lo ve, entonces, como ella tiene el libro de texto de la $\mathrm{ONCE},{ }^{2}$ si utilizo el libro de texto ella puede seguir lo que yo voy escribiendo en la pizarra y estos dibujos hacérselos en papel como lo hacéis vosotros.

En este ejemplo, la dificultad para el aprendizaje del concepto matemático determinado con la estudiante con deficiencia visual deviene en un conocimiento de necesidades específicas debidas a las características de aprendizaje del caso particular. En este sentido, la necesidad procede de una dificultad. Sin embargo, no todo el conocimiento de necesidades estará relacionado con dificultades de aprendizaje. Por ejemplo, el conocimiento que tenga el profesor sobre qué contenidos requiere saber el estudiante para enfrentarse a un tema nuevo puede venir de las fortalezas que esa red de conexiones matemáticas promueven en el aprendizaje de los estudiantes (Silverman y Thompson, 2008). Aunque inicialmente el siguiente indicador surge a partir de una estudiante con deficiencia visual severa, ${ }^{3}$ a través de varios estudios (Ruiz y Sosa, 2011; Martínez y Sosa, 2012) hemos podido notar la relevancia de este indicador incluso con estudiantes de capacidades medias, haciendo énfasis en la importancia de que el profesor sepa los conocimientos matemáticos previos que necesita el estudiante para poder lograr el aprendizaje de un contenido matemático específico. De lo anterior resulta el indicador:

2. La ONCE es una asociación de discapacitados, incluyendo a las personas que tienen deficiencia visual, que ofrece a los estudiantes el libro de texto que llevan en el instituto pero en código Braille.

3. Lo cual marca un reto más para el profesor en cuanto al material que debe utilizar, así como la búsqueda de un adecuado contexto comunicacional que se adapte a las necesidades de dichos estudiantes (Camargo y Nardi, 2013). 
KFLM4. Saber las necesidades y dificultades de los estudiantes sobre el contenido matemático.

En los siguientes dos episodios queremos hacer notar varios aspectos concretos de los conocimientos del profesor en cuanto a confusiones matemáticas que pudieran tener los estudiantes.

En la octava clase grabada, Aly evidencia que sabe que los estudiantes se pueden equivocar cuando haya un número real multiplicando al determinante al confundirse con el producto de un escalar por una matriz. Antes dejó de deberes calcular determinantes de orden mayor que tres. Al empezar la clase Aly empieza a resolverlos en la pizarra. Uno de los incisos consiste en calcular $\left(\begin{array}{ccc}5 x & 5 y & 5 \\ 1 & 0 & \frac{3}{5} \\ 1 & 1 & 1\end{array}\right)$ sabiendo que $\left(\begin{array}{lll}x & y & z \\ 5 & 0 & 3 \\ 1 & 1 & 1\end{array}\right)=1$

Hasta el momento, usando una propiedad de los determinantes, han sacado el 5 de la primera fila del determinante, obteniendo $5\left(\begin{array}{ccc}x & y & z \\ 1 & 0 & \frac{3}{5} \\ 1 & 1 & 1\end{array}\right)$, luego Aly comenta:

Bueno, de la misma manera que la propiedad dice que un número se puede sacar fuera de un determinante, la misma propiedad me dice que un número que está fuera multiplicando el determinante ¿̇cómo lo puedo expresar? [Aly señala la primera fila $5 x, 5 y, 5 z$ ], multiplicando a la fila que a mí me dé la gana, a una fila [Aly pronuncia más fuerte la palabra una] o a una columna, ¿de acuerdo?

Para el siguiente paso, Aly remarca que al introducir el 5 al determinante tiene que ser multiplicado solo por una fila o una columna. Esto considerando que antes vieron la multiplicación de un escalar por una matriz y, como Aly confirma en la entrevista, sabe que los estudiantes pueden confundir el producto de un escalar por un determinante con el producto de un escalar por una matriz. En este sentido queremos poner de relieve el conocimiento del profesor referente a las confusiones matemáticas que pudiera tener el estudiante, provocadas por la relación equivocada de un contenido actual con un contenido relativamente anterior.

En álgebra, hay varios temas que aparecen en el currículum para ser enseñados que toman la forma de reglas o algoritmos. Estos temas, en términos de aprendizaje, pueden contribuir, per se, a la mecanización y confusión más que al significado del concepto. En el aula, esto suele tener fuertes implicaciones cuando aquellos temas que deben ser enseńados en la misma unidad o bloque son especialmente susceptibles de causar confusiones por similitud. En este sentido proponemos el indicador:

KFLM5. Conocer las confusiones matemáticas que pudiera tener el estudiante, provocadas por la relación equivocada de un contenido actual con un contenido relativamente anterior.

En el siguiente episodio Emi está explicando cómo utilizar el método de reducción de Gauss para resolver sistemas de ecuaciones lineales; después de diagonalizar la matriz ampliada (matriz de coeficientes con adhesión de la matriz de términos independientes) y escribir el sistema equivalente, les remarca:

Fijaos que he escrito el sistema equivalente poniendo siempre las incógnitas por columnas, las equis debajo de las equis $\left[x^{\prime} s\right]$, las y's debajo de las y’s y las z’s debajo de las z’s [...] ¿Cuál es la solución entonces? La solución es $x$ vale $1600, y$ vale 1000 y $z$ vale 3000 . Es así como se escribe la solución a un sistema [Emi se refiere a la notación "solución $(x=1600, y=1000, z=3000)$ »]. Si yo lo escribo en otro orden, no es que esté incorrecto, pero siempre se ordenan las incógnitas en el orden en que aparecen dadas. 
Queremos destacar el conocimiento que pone en acción Emi respecto a saber que los estudiantes pueden cometer un error referente al adecuado acomodo de los elementos en una matriz con base en su posición y al momento de escribir la solución del sistema sin seguir la convención matemática de anotar el valor de las incógnitas en el orden en que aparecen dadas, en este caso $(x, y, z)$. Por eso, proponemos el indicador:

KFLM6. Conocer las confusiones y los errores matemáticos de los estudiantes, producidos por no proceder ordenadamente, o no respetar las convenciones matemáticas.

Hay investigaciones que reportan elementos considerados por algunos profesores referentes al manejo del error y las confusiones de los alumnos (Ma, 2010). En este caso las evidencias nos permiten dar cuenta de aspectos más específicos del conocimiento del profesor en cuanto a confusiones y errores matemáticos, por ello la propuesta de estos dos últimos indicadores.

En la clase grabada 7 de Aly, tras ver que cuando por debajo de la diagonal principal todos los elementos son cero el determinante de esa matriz dará como resultado el producto de los elementos de la diagonal principal, Aly les pregunta cuánto daría el determinante de la matriz en la cual debajo de la diagonal secundaria todos fueran cero; un estudiante contesta «menos», y ella les comenta que en efecto dará como resultado el producto de esa diagonal secundaria precedida por el signo menos, y luego les aclara lo siguiente:

Cuando digo que sale negativo [Aly señala el menos que precede al resultado del determinante], quiere decir que cambia de signo, pues puede ser que si el producto me sale negativo, con el menos por delante, saldría al final el resultado positivo.

Queremos destacar que Aly sabe que, al haber dicho «negativo», los estudiantes se pueden quedar con la imagen inadecuada de que en ese tipo de matrices siempre va a quedar en el resultado un número negativo. En este sentido, queremos destacar el conocimiento del profesor en cuanto a las imágenes o ideas matemáticas inadecuadas que los estudiantes pueden poseer o adquirir de un contenido matemático, obtenidas a partir de imágenes e ideas ya hechas previamente en la mente del estudiante, o bien aquellas que puedan ser producidas por el propio discurso matemático que utiliza el profesor al impartir un contenido. Es importante que el profesor conozca cómo podrían llegar a pensar los estudiantes el concepto matemático y cómo están facultados para aprender otras ideas matemáticas relacionadas (Silverman y Thompson, 2008). Por tanto proponemos el indicador:

KFLM7. Conocerlas imágenes o ideas matemáticas inadecuadas que los estudiantes pueden poseer o adquirir de un contenido.

En la tercera clase grabada de Emi, una estudiante le comenta que a ella no le coincide el número que Emi escribió en la tercera fila primera columna del resultado del producto de dos matrices. Emi señala en la pizarra el elemento correspondiente a esa indicación y les menciona que ese elemento viene de multiplicar la tercera fila por la primera columna, además de explicitar cada uno de los productos involucrados. Luego les comenta:

Aquí las equivocaciones pueden producirse por dos motivos, uno que multipliquemos mal o sumemos mal [...] entonces aquí hay que estar atentos a las cuentas con los números y también hay que estar atentos a la geometría del producto, esto tiene una geometría y tenemos que ir en un orden determinado.

Sobre esto, queremos poner de relieve el conocimiento de la profesora de los errores que los estudiantes pueden cometer al realizar el producto de dos matrices al hacer mal algún cálculo provocado por un despiste, sobre todo al llevar a cabo una cadena de operaciones aritméticas mentalmente, o al ejecutar esas operaciones sin dominar aún el nuevo contenido que se está abordando. El conocimiento del profesor referente a identificar y proporcionar explicaciones sobre errores y conocer qué errores se presentan con qué contenido, así como conocer las estrategias comunes de cálculo de los estudiantes 
(familiarización con los números y las operaciones), son dos de las cuatro categorías abordadas por Hill et al. (2008). Basándonos en las evidencias presentamos un indicador enfocado en elementos concretos del conocimiento del profesor referente a aspectos en los que los estudiantes son más proclives a cometer errores en determinados cálculos aritméticos.

KFLM8. Conocer los errores que los estudiantes pueden cometer al hacer determinados cálculos aritméticos provocados por un despiste al hacer operaciones o transformaciones, o por no dominar el nuevo contenido que se está abordando.

En el siguiente episodio queremos destacar que Emi, prevé que los estudiantes no se percaten de que un problema es análogo a otro que han visto anteriormente. En clases pasadas, Emi hizo un ejemplo de un problema de programación lineal similar al ejercicio que están haciendo en la clase 13; al ver que los estudiantes no hacen el ejercicio, les comenta:

¿No les recuerda este problema a uno que ya habíamos planteado? El de la fábrica de refrescos y los supermercados, ¿cuántas fábricas de refrescos teníamos? Dos fábricas, y supermercados a donde se distribuían, tres, ¿̇no? y ¿qué nos daban?

Emi sabe que los estudiantes tienen dificultades para resolver un problema y sabe que si ven que dicho problema es parecido a un ejemplo realizado antes, entonces podrán hacer una analogía y lograr resolverlo. De esta manera, proponemos el indicador:

KFLM9. Conocer que los estudiantes tienen dificultades en reconocer y aplicar analogías y equivalencias en la resolución de problemas.

Existen investigaciones que dan cabida al conocimiento del profesor en cuanto a errores y dificultades de los estudiantes (Rowland et al., 2005), e incluso explicitan el manejo del error por parte del profesor (Ma, 2010). En los indicadores desde el KFLM4 hasta el KFLM9 hemos destacado aspectos que detallan y matizan el conocimiento del profesor en torno a necesidades, dificultades, confusiones, imágenes e ideas inadecuadas y errores. Estos aspectos se pueden integrar en una categoría que hemos denominado errores y dificultades asociadas al aprendizaje.

En la clase grabada número 4 de Emi, ella sabe que habrá algún estudiante que no sepa en qué consiste la propiedad conmutativa del producto de números reales. A Emi le interesa repasar esa propiedad para hacer notar que en el producto de matrices no se cumple.

Emi Hay una propiedad de los números que no se cumple en las matrices, ¿cuál es? Sabéis cuál es la propiedad conmutativa en el producto, igual que para la suma, pero ahora para el producto, el orden en que se efectúe un producto con los números reales no importa, lo mismo es multiplicar $a$ por $b$ que $b$ por $a$. ¿Va a cumplirse esto con las matrices?

E4: No.

Emi: Pues no, es decir, $A$ por $B$ va a ser distinto de $B$ por $A$. En este caso la propiedad conmutativa no se cumple, en general $A$ por $B$ va a ser distinto de $B$ por $A$.

Podemos interpretar que Emi sabe que en el aprendizaje de ese contenido matemático puede ser de ayuda aprovechar lo que el estudiante ya conoce y que, por tanto, es importante afianzar ese contenido matemático previo y aprovecharlo para presentarles el nuevo contenido. De esa manera proponemos el indicador:

KFLM10. Conocer los contenidos matemáticos previos de los que se puede valer para fomentar el aprendizaje de un tema nuevo entre sus estudiantes.

En este episodio llama la atención en primer término el conocimiento que tiene la profesora acerca de que el producto de matrices no es conmutativo, lo cual forma parte de su conocimiento matemático. También podemos ver que ella sabe que los estudiantes suelen extrapolar propiedades de los números reales a las matrices. Pero sobre todo esto queremos destacar el uso que hace de los conocimientos 
previos, que en forma parecen equivalentes a los que está enseñando. Este uso involucra conocimiento de la profesora acerca del error en matemáticas (uso con el que podemos o no coincidir), de la anticipación a los modos de pensamiento de los estudiantes y de cómo negociar significados (Godino, 2009) con los estudiantes (de nuevo podemos o no coincidir con la forma de negociación), que en su conjunto nos ofrece información acerca de elementos de una teoría personal sobre el aprendizaje de las matemáticas.

Es por eso que este indicador nos inspira a conformar la categoría teorías personales sobre formas de aprendizaje. Deseamos poner de relieve que el hecho de que el profesor abstraiga de qué manera es conveniente aprovechar el conocimiento matemático que posee el estudiante para explicar el nuevo contenido es un indicio de una conceptualización personal acerca de cómo se puede aprender matemáticas. La reiteración de la profesora al aludir a la propiedad conmutativa de los números reales para señalar la importancia de no extrapolar directamente las propiedades conocidas hacia las operaciones con matrices nos habla de la forma en que ella concibe que aprenderán esas nuevas propiedades. Más aún, eso lo usa con doble intención en el aprendizaje y la enseñanza, pues además de aprovechar el conocimiento matemático que poseen algunos estudiantes (inclusive hacerles algunas rectificaciones) socializa este en el grupo, pensando en aquellos estudiantes que no conozcan ese contenido matemático previo.

Tabla 1.

Categorías e indicadores obtenidos para el KFLM

\begin{tabular}{|c|c|}
\hline Categoría & Indicador \\
\hline \multirow[t]{3}{*}{$\begin{array}{l}\text { a) Lenguaje y procesos con los que los } \\
\text { estudiantes interactúan con el con- } \\
\text { tenido }\end{array}$} & $\begin{array}{l}\text { KFLM1. Saber interpretar el conocimiento o pensamiento matemático que expre- } \\
\text { san los estudiantes en su lenguaje (común o en proceso de adquisición del nuevo } \\
\text { contenido matemático - mezcla del lenguaje común con el matemático-). }\end{array}$ \\
\hline & $\begin{array}{l}\text { KFLM2. Conocer los detalles de la resolución de un problema susceptibles de des- } \\
\text { viar la atención de los estudiantes para llegar a su solución. }\end{array}$ \\
\hline & $\begin{array}{l}\text { KFLM3. Conocer los cálculos matemáticos que podrían realizar de forma mecánica } \\
\text { los estudiantes sin saber en realidad lo que están haciendo matemáticamente. }\end{array}$ \\
\hline \multirow[t]{6}{*}{$\begin{array}{l}\text { b) Errores y dificultades asociadas al } \\
\text { aprendizaje }\end{array}$} & $\begin{array}{l}\text { KFLM4. Conocer las necesidades y dificultades de los estudiantes sobre el conte- } \\
\text { nido matemático. }\end{array}$ \\
\hline & $\begin{array}{l}\text { KFLM5. Conocer las confusiones matemáticas que pudiera tener el estudiante, } \\
\text { provocadas por la relación equivocada de un contenido actual con un contenido } \\
\text { relativamente anterior (por ejemplo con un tema pasado de la misma unidad o } \\
\text { bloque temático). }\end{array}$ \\
\hline & $\begin{array}{l}\text { KFLM6. Conocer las confusiones y los errores matemáticos de los estudiantes, pro- } \\
\text { ducidos por no proceder ordenadamente o no respetar las convenciones matemá- } \\
\text { ticas. }\end{array}$ \\
\hline & $\begin{array}{l}\text { KFLM7. Conocer las imágenes o ideas matemáticas inadecuadas que los estudian- } \\
\text { tes pueden poseer o adquirir de un contenido. }\end{array}$ \\
\hline & $\begin{array}{l}\text { KFLM8. Conocer los errores que los estudiantes pueden cometer al hacer deter- } \\
\text { minados cálculos aritméticos provocados por un despiste al hacer operaciones o } \\
\text { transformaciones, o por no dominar el nuevo contenido que se está abordando. }\end{array}$ \\
\hline & $\begin{array}{l}\text { KFLM9. Conocer que los estudiantes tienen dificultades en reconocer y aplicar } \\
\text { analogías y equivalencias en la resolución de problemas. }\end{array}$ \\
\hline $\begin{array}{l}\text { c) Teorías personales sobre formas de } \\
\text { aprendizaje }\end{array}$ & $\begin{array}{l}\text { KFLM10. Conocer los contenidos matemáticos previos de los que se puede valer } \\
\text { para fomentar el aprendizaje de un tema nuevo entre sus estudiantes. }\end{array}$ \\
\hline
\end{tabular}




\section{CONCLUSIONES}

Es importante que el profesor conozca cómo aprenden sus estudiantes cada contenido que desea impartir (Sosa, 2012). Consideramos que el KFLM puede ayudar al profesor a impartir una enseñanza más efectiva fomentando el aprendizaje matemático del estudiante. Si realmente queremos entender y comprender el conocimiento del profesor en cuanto a saber cómo pueden pensar o aprender las matemáticas los estudiantes, no basta con establecer y definir un subdominio, es conveniente indagar en la profundización de este, saber de manera más precisa cuáles son las características de aprendizaje, cómo y de dónde surgen, y cuál es la interacción de los estudiantes con ese contenido matemático durante el aprendizaje de este. Indiscutiblemente establecer categorías tanto desde la literatura como desde las evidencias empíricas nos permite acercarnos más a describir indicadores que den cuenta de esas categorías o aspectos relevantes que se van deduciendo. Resulta interesante remarcar la importancia no solo de considerar las categorías que pudiéramos deducir de la literatura sino contrastarlas con las evidencias suscitadas directamente en la práctica del profesor. Para poder llevar a cabo esa profundización son sumamente importantes tanto las categorías como los indicadores. Ambos tienen un papel y una riqueza por sí mismos. Por un lado, los indicadores nos permiten tener mayor especificidad del conocimiento del profesor en cuanto a aspectos o rasgos de los procesos por los que el estudiante transita para aprehender objetos matemáticos concretos así como de los fenómenos derivados de esto. Por otra parte, las categorías nos permiten agrupar aquellos aspectos o elementos que pudieran ser comunes entre indicadores o que tengan una naturaleza similar entre ellos e identificar campos amplios de intervención para la formación del profesorado a este respecto.

En este artículo solo mostramos un caso particular del KFLM, pero seguimos trabajando en la profundización no solo de estas categorías y de este subdominio sino también de los otros subdominios del MTSK en diferentes contenidos matemáticos y niveles educativos. Entendemos que analizar en profundidad los subdominios del MTSK a través de distintos matices (por ejemplo, categorías e indicadores) puede contribuir a la identificación, comprensión y análisis del conocimiento didáctico del contenido de otros profesores y del conocimiento disciplinar, los cuales pueden ser tomados en cuenta en la formación inicial y continua del profesor así como para o por formadores de profesores (Sosa y Ribeiro, 2014).

Finalmente queremos destacar que estudios como el nuestro pueden contribuir a la vinculación investigación-práctica e incluso resultar relevantes de una forma directa en la enseñanza y en la conceptualización de la práctica del profesor. Las categorías e indicadores pueden servir como una fuente para el trabajo de enseñanza. Sin embargo, aún faltan estudios sobre cómo la investigación sobre el conocimiento del profesor puede afectar a la práctica, además de otras investigaciones que den cuenta de la relación que guardan estas y otras categorías y sus respectivos indicadores, en términos de la planificación, implementación y reflexión de la enseñanza; así como el impacto de ese conocimiento del profesor en el aprendizaje de los estudiantes y cómo el conocimiento propicia interacciones en el aula.

\section{AGRADECIMIENTOS}

Esta investigación es apoyada por el Consejo Nacional de Ciencia y Tecnología y la Secretaría de Educación Pública de México, y por el Ministerio de Economía y Competitividad de Espańa mediante el proyecto de investigación «Caracterización del conocimiento especializado del profesorado de Matemáticas» (EDU2013-44047-P). 


\section{REFERENCIAS BIBLIOGRÁFICAS}

Bisquerra, R. (2004). Metodología de la investigación educativa. Madrid, España: La Muralla.

Camargo, E. P. y Nardi, R. (2013). Contextos comunicacionales adecuados e inadecuados para la inclusión de alumnos con discapacidad visual en clases de física moderna. Enseñanza de las Ciencias, 31(3), pp. 155-175.

Carrillo, J.; Climent, N.; Contreras, L. C. y Muñoz-Catalán, M. C. (2013). Determining specialised knowledge for mathematics teaching. En B. Ubuz, C. Haser y M. A. Mariotti (eds.). Proceedings of the CERME 8. Middle East Technical Universitiy, Ankara, Turquía: ERME, pp. 2985-2994.

Cohen L. y Manion L. (2002). Métodos de investigación educativa. Madrid: La Muralla.

Even, R. y Markovits, Z. (1991). Teachers' pedagogical knowledge: The case of functions. En F. Furinghetti (ed.). Proceedings of the $15^{\text {th }}$ PME International Conference, 2, pp. 40-47.

Evertson, C. M. y Green, J. L. (1989). La observación como indagación y método. En C. M. Wittrock (ed.). La investigación de la enseñanza, II: Métodos cualitativos y de observación. Barcelona: Paidós/MEC, pp. 303-406.

Flores-Medrano, E.; Escudero-Avila, D.; Montes, M. y Carrillo, J. (2014). ¿Cómo se relaciona el conocimiento que tiene el profesor acerca del aprendizaje de las matemáticas con su entendimiento sobre los espacios de trabajo matemático? Manuscrito sometido a evaluación.

Gess-Newsome, J. (1999). Introduction and orientation to examining pedagogical content knowledge. En J. Gess-Newsome y N. G. Lederman (eds.). Examining pedagogical content knowledge. Dordrecht, The Netherlands: Kluwer Academic Publishers, pp. 3-20.

Godino, J. D. (2009). Categorías de análisis de los conocimientos del profesor de matemáticas. Unión. Revista Iberoamericana de Educación Matemática, 20, pp. 13-31.

Hill, H.; Ball, D. y Schilling, G. (2008). Unpacking pedagogical content knowledge: conceptualizing and measuring teachers' topic-specific knowledge of students. Journal for Research in Mathematics Education, 39(4), pp. 372-400.

Kilpatrick, J.; Swafford, J. y Findell, B. (2001). Adding it up. Washington: National Academy Press.

Kuzniak, A. (2011). L'espace de Travail Mathématique et sesgenèses. Annales de didactique et de sciences cognitives, 16, pp. 9-24.

Latorre, A.; Rincón, D. del. y Arnal, J. (1996). Bases metodológicas de la investigación educativa. Barcelona: Hurtado ediciones.

Lofland, J. y Lofland, L. H. (1984). Analyzing social settings. Belmont, CA: Wadsworth Publishing Company, Inc.

MA, L. (2010). Conocimiento y enseñanza de las matemáticas elementales. Chile: Academia Chilena de Ciencias.

Martínez, C. V. y Sosa, L. (2012). Conocimiento del profesor acerca de las dificultades que enfrentan sus estudiantes al cursar el tema sistema de ecuaciones lineales en bachillerato. Un estudio de caso. En L. Sosa, E. Aparicio y F. M. Rodríguez (eds.). Memorias de la XV Escuela de Invierno en Matemática Educativa. México, D. F., pp. 21-25.

Merriam, S. B. (1988). Case Study Research in Education: A qualitative approach. San Francisco, CA: Jossey-Bass Publishers.

Pinto, J. y González, M. T. (2006). Sobre la naturaleza conceptual y metodológica del conocimiento del contenido pedagógico en matemáticas. Una aproximación para su estudio. En M. P. Bolea, M. Moreno y M. J. González (eds.). Investigación en Educación Matemática X. Huesca: SEIEM, pp. 237-255. 
Ponte, J. P. y Chapman, O. (2006). Mathematics teachers' knowledge and practice. En A. Gutierrez y P. Boero (eds.). Handbook of Research of the Psychology of Mathematics Education: Past, Present and Future. Rotterdam: Sense Publishing, pp. 461-494.

Rowland, T.; Huckstep, P. y Thwaites, A. (2005). Elementary teachers' mathematics subject knowledge: The knowledge quartet and the case of Naomi. Journal of Mathematics Teacher Education, 8(3), pp. 255-281.

http://dx.doi.org/10.1007/s10857-005-0853-5

Ruiz, G. y Sosa, L. (2011). Conocimiento del profesor en cuanto a conocimiento del contenido y estudiantes en el tema de operaciones fundamentales con expresiones algebraicas en bachillerato. En L. Sosa, R. Rodríguez y E. Aparicio (eds.). Memorias de la XIV Escuela de Invierno en Matemática Educativa. México, D. F., pp. 9-15.

Shulman, L. S. (1986). Those who understand: knowledge growth in teaching. American Educational Research Association, 15(2), pp. 4-14.

http://dx.doi.org/10.3102/0013189X015002004

Silverman, J. y Thompson, P. W. (2008). Toward a framework for the development of mathematical knowledge for teaching. Journal of Mathematics Teacher Education, 11(6), pp. 499-511. http://dx.doi.org/10.1007/s10857-008-9089-5

Sosa, L. (2012).Conocimiento del profesor para la enseñanza de las matemáticas. Contribución teórica al conocimiento del contenido y estudiantes. Acta Latinoamericana de Matemática Educativa, vol. 25, pp. 1151-1159.

Sosa, L. y Ribeiro, C. M. (2014). La formación del profesorado de matemáticas de nivel medio superior en México: una necesidad para la profesionalización docente. Revista Iberoamericana de Producción Académica y Gestión Educativa, 1, pp. 1-15.

Stake, R. E. (1994). Case Studies. En N. K. Denzin y Y. S. Lincoln (eds.). Handbook of Qualitative Research. Thousands Oaks, CA: Sage Publications, pp. 236-247.

Tatto, M. T.; Schwille, J.; Senk, S. L.; Ingvarson, L.; Peck, R. y Rowley, G. (2008). Teacher education and development study in mathematics (TEDS-M): Conceptual framework. East Lansing, MI: Teacher Education and Development International Study Center, College of Education, Michigan State University.

Varas, L.; Lacourly, N.; López, A. y Giaconi, V. (2013). Evaluación del conocimiento pedagógico del contenido para enseñar matemáticas elementales. Enseñanza de las Ciencias, 31(1), pp. 171187.

YIN, R. (1984). Case study research. Design and methods. Newbury Park, CA: Sage. 


\title{
Teacher Knowledge about the features of learning algebra in high school
}

\author{
Leticia Sosa \\ Universidad Autónoma de Zacatecas \\ Isosa@mate.reduaz.mx \\ Eric Flores-Medrano, José Carrillo \\ Centro de Investigación en didácticas específicas e investigación en el aula (CIDIESIA), Universidad de Huelva \\ ericfm_0@hotmail.com, carrillo@uhu.es
}

This is a study in the field of research into Mathematics Teachers' Knowledge and Professional Development. The theoretical background to the study makes use of the model of teachers' knowledge known as Mathematics Teacher's Specialised Knowledge. This model recognises three sub-domains of mathematical knowledge (Knowledge of Topics, Knowledge of the Structure of Mathematics, and Knowledge of Practices in Mathematics) and three with respect to Pedagogical Content Knowledge (Knowledge of Mathematics Teaching, Knowledge of the Features of Learning Mathematics, and Knowledge of Mathematics Learning Standards). This study delves deeper into the understanding of the elements contributing to Knowledge of the Features of Learning Mathematics (KFLM).

Two teachers were selected, both teaching Linear Algebra for the Spanish Baccalaureate (ages 16-18), one in the Science and Technology branch, the other in the Social Science branch. The research design involved observing each teacher over the course of fifteen 50 -minute lessons, adhering to a strict non-interventionist policy. The lessons were video-recorded and field notes were taken so as to provide support for the transcribed material. This work was followed up with semi-structured interviews examining specific points of interest.

Various episodes were selected and analysed for aspects of the teachers' knowledge of their students' learning process using knowledge indicators (which emerged from literature revision) from the relevant sub-domain of the theoretical construct employed for the purpose (described in section on theoretical background).

The category Language and processes by which students interact with content arose from consideration of three indicators (which emerged from the analysis): (a) demonstrating understanding of students' forms of expressing mathematical knowledge or thinking; (b) demonstrating awareness of those elements of working through a problem likely to distract students from arriving at a solution; and (c) demonstrating awareness of mathematical calculations which students can do mechanically without understanding the underlying mathematics.

In like fashion, the category Errors and diffculties associated with learning derived from the following six indicators: (a) demonstrating knowledge of students' needs and diffculties in relation to mathematical content, (b) demonstrating understanding of potential student misunderstandings arising from the confusion of current content with previous content, (c) demonstrating understanding of student misunderstandings and errors arising from the failure to apply procedures in an orderly fashion or to observe mathematical conventions, (d) demonstrating understanding of inadequate mathematical images or ideas which students might have or acquire about a content, (e) demonstrating understanding of potential student errors in arithmetic calculations arising from distractions when doing operations or transformations, or from not being able to manage the new content in question, and (f) demonstrating awareness of student difficulties in recognising and applying analogies and equivalences in problem solving.

Finally, the category Personal theories of learning was established on the evidence of a strategy aimed at generating a mathematically edifying task among the students.

We hope that the study contributes to the link between research and practice in terms of developing our understanding of teachers' knowledge of the process of learning mathematics. 
